\title{
The Senescent Lens and the Retinal Pigment Epithelium
}

\author{
R A WEALE
}

London

Lipofuscin is a light-absorbing intra-cellular inclusion accumulating with age in a number of tissues such as human sympathetic ganglia, ${ }^{\prime}$ uterus, duodenum, ${ }^{2}$ and the retinal pigment epithelium or RPE. ${ }^{3,4}$ This is why it is numbered amongst the ageing pigments. Whereas its increase in the rat RPE is approximately linear ${ }^{5}$ for at least a fair proportion of the animal's lifespan, ${ }^{6}$ this is not so in man. ${ }^{3.4}$ It builds up mainly during the first two or three decades of life, but a second phase of increase begins after the age of about 50 years. Its build-up has been correlated also with stress, ${ }^{7}$ a variety of metabolic disturbances ${ }^{8}$ and deficiencies, ${ }^{2}$ but the view that its formation is necessarily detrimental to cellular function has been challenged. ${ }^{9}$

The accumulation of lipofuscin in the youthful human RPE does not tally with the notion that we are dealing exclusively with a sign of ageing, and it has therefore been attributed to retinal exposure of radiations of short wavelengths. ${ }^{10,11}$ On this view it accretes following retinal irradiation in the range of $295-400 \mathrm{~nm}$, the rate declining in line with the increase in the ultraviolet absorbance of the crystalline lens with age "on a time scale that matches" the RPE lipofuscin build-up. ${ }^{12}$

There is also a spatial distribution of lipofuscin across the retina, ${ }^{4}$ with a dip in the macular region at the posterior pole of the eye attributed ${ }^{10}$ to the absorbing, and perhaps protective, properties of the yellow macular pigment.

However, the implicit quantitative relations between radiation and the build-up of lipofuscin in the retinal pigment epithelium have not been scrutinised with the rigour that might enable one to verify some of the hypotheses postulated to underlie them. Moreover, even the previous qualitative argument is partly incomplete and partly defective. As the implicit misunderstanding of photogiological processes does not seem to be isolated, ${ }^{13}$ it may be helpful to illustrate some principles relevant to a few of the interdisciplinary studies now being carried out and to examine how they can be applied to potential relations between radiation and lipofuscin buildup in the human retinal pigment epithelium.

Attention needs to be drawn to variables that have been overlooked in the past, and which may enable one to make some tentative choices between competing hypotheses.

\section{Theory}

The lenticular absorbance data quoted ${ }^{12}$ fail to show any variation with age above 25 years in the spectral range of $295-400 \mathrm{~nm}$, and have been overtaken by others from that source, ${ }^{14,15}$ similarly invariant. A more detailed study ${ }^{16}$ of human lenticular absorbance $\mathrm{D}$ has revealed an age variation between at least 330 and $600 \mathrm{~nm}$, enabling one to predict $D$ from a simple exponential relation, namely

$\mathrm{D}(\mathrm{t})=\mathrm{D}(0) \exp (\beta \mathrm{t})$

where $\mathrm{D}(0)$ is the absorbance at birth, $\beta$ a coefficient of the order of 0.02 , and the age in years. $\mathrm{D}(0)$ varies greatly with the wavelength $\lambda$, $\beta$ does so to a smaller degree.

Now retinal illumination does not vary with age only because of lenticular absorbance. Numerous studies (cf. 17) have shown that the pupillary area $\mathrm{P}(\mathrm{t})$ declines after attaining a peak in the lower teens, thereby reducing the amount of radiation of all wavelengths reaching the retina. This senile miosis is not trivial, the pupillary area in one's sixties being approximately one third the value at twenty years of age: 
the ensuing loss of light is therefore of the same order as that due to the change in the u.v. absorbance of the lens.

Again, in everyday life, our eyes are not exposed just to an arbitrarily selected wavelength range but to a wide spectrum, namely that characteristic of daylight. Although this can vary, "average" daylight has been defined, and quantified by the International Commission for Illumination as Standard B or S(B): it peaks mildly in the blue part of the spectrum. ${ }^{18}$

Also for radiation to act, it must do more than impinge on the retina: it must be absorbed. Although lipofuscin is a pigment it cannot be a candidate as an absorber since one postulates that, in some way, photic action produces it. If it accumulates owing to radiation being absorbed by the retinal photo-receptors, rods or cones or both, during the normal process of vision, the relevant visual pigment absorption characteristics have to be considered. To a first approximation the relevant sensitivity function $F(\lambda)$ is given by the $\mathrm{V}(\lambda)$ curve, ${ }^{17}$ used for describing spectral aspects of daylight vision: the $V^{\prime}(\lambda)$ curve provides analogous data for rods and twilight vision.

If one were dealing with almost monochromatic light, the next step would be the simple one employed earlier ${ }^{4,10}$ when a relatively small part of the u.v. spectrum was deemed to cause the build-up of lipofuscin in the human RPE. The radiation giving rise to the response might be thought of as proportional to the product of irradiance B (as modified by intervening filters) and sensor sensitivity. Then

$R(t)=k \cdot B(\lambda) \cdot F(\lambda) \cdot f \cdot T(\lambda) \cdot \int P(t) \cdot T(t, \lambda) \cdot d t$

where $R$ is the quantity of lipofuscin accumulated at age $t, k$ a constant, $f$ the fraction of sensors with sensitivity $F(\lambda), T(\lambda)$ the transmissivity of invariant filters such as the macular pigment, ${ }^{19}$ $P$ the pupillary area at age $t$, and $T(t, \lambda)$ the lenticular transmissivity, the other symbols being as previously defined.

The real situation is, however, different as noted above; and it is therefore necessary to weight the overall active stimulus by adding together all the above (non-zero) products for narrow wavelength ranges, or, to use a technical term, to convolute radiation with transmissivity and sensor sensitivity.

Although open to some criticism, the value for $f$ can be obtained from conventional data. ${ }^{20}$ The

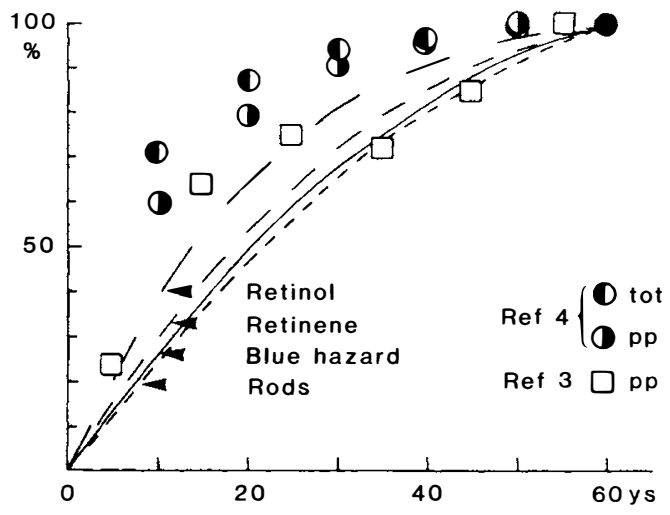

Fig. 1: Comparison of experimental data ${ }^{3,4}$ and calculated radiation cumulants for different ages. Parameters: absorbers of radiation. tot $=$ total RPE; $p p=$ posterior pole .

above-mentioned convolution then gives

$R(\lambda, t)=k \cdot \iint B(\lambda) \cdot F(\lambda) \cdot f \cdot T(\lambda) \cdot P(t) \cdot T(\lambda, t) \cdot d \lambda \cdot d t \cdot(3)$ $\mathrm{T}(\lambda, \mathrm{t})$ is related to $\mathrm{D}$ in eq. $(1)$ by $\mathrm{T}(\lambda, \mathrm{t})=10^{-\mathrm{D}(\lambda, \mathrm{t})}$.

\section{Validation}

Figure 1 shows the results of two studies ${ }^{3,4}$ with data up to and including the sixth decade of life, where they are normalised. In both, results were obtained for even higher age-groups, but these are ignored here as the focus is on the early rise in lipofuscin.

The substitution of numerical values ${ }^{16,20}$ enables one to test a variety of hypotheses with the help of eq. 3. This requires both sides of the equation to be normalised: since, by definition, the response at birth must be zero, but irradiation is not, it is convenient to plot and to normalise changes in retinal irradiation.

The data represent the left-hand side of eq. 3: how is one to compare them with the right-hand one? Fitting a curve depends crucially on a normalised value for this product. Much could be achieved if one could follow earlier authors in their tacit assumption that the radiation is virtually monochromatic. Now the right-hand function does not reach a plateau similar to that indicated by $R$, even though its initial rise is greater than any other. This is where the similarity between the two functions ends. $R$ rises initially more steeply than any of the following postulated functions. (Fig. 1). 
(a) Substituting the cone-function $V(\lambda)$ for $F(\lambda)$ produces a relatively slowly rising curve, because lens absorption affects it relatively weakly. In fact, the calculated function barely differs from one computed on the assumption that the only senescent change to occur is senile miosis. If there were no change even in pupillary area, the rise would be, of course, rectilinear.

(b) The rod-curve $V^{\prime}(\lambda)$ naturally fares better, because it occupies a shorter wavelength range, but its rise still lags behind that of $\mathrm{R}$.

(c) The risk function for the so-called "blue hazard" 21 also reduces the difference between the two, but still insufficiently.

(d) It is possible to speculate on an $F(\lambda)$ function which might yield the initially rapid rise that is required to satisfy the hypothesis; retinol[1] or perhaps retinol might fit the bill, but may conflict with an additional requirement, namely a consistent explanation of the variation of $R$ across the retina (Fig. 2).

However, Katz et al..$^{22}$ have recently shown that, in the case of rat retinae, lipofuscin accumulates as a result of the operation of two mechanisms, namely autophagy and heterophagy. If radiation is an active agent in the formation of lipofuscin it may act both directly on vitamin A and via the photolysis of the photo-receptors. As yet there is no evidence (i) that this happens, and (ii) that it happens in the human retina. But it may be noted that with half-times of 20 years for the rod (Fig.1) and 13 for the retinol curves respectively, their combined effect leads to a value of eight years, which is consistent with observation.

Figure 2 raises the question whether both rods and cones contribute to the formation of lipofuscin. It shows a comparison between the mean spatial lipofuscin distribution ${ }^{4}$ across the retina and the flux density of violet light; but for the intervention of the optic disc, the curves would continue almost symmetrically about the fovea on the nasal side. Note that the pronounced dip in the curve for the violet light is due to the absorption of the macular pigment. Its overall effect must be diminished in average day-light (Fig.2b), when the vertical bar delineates the extremes of the caculated dip, the curve for the youngest group lying at the bottom of the bar: the rest of the (day-light) curve has been omitted
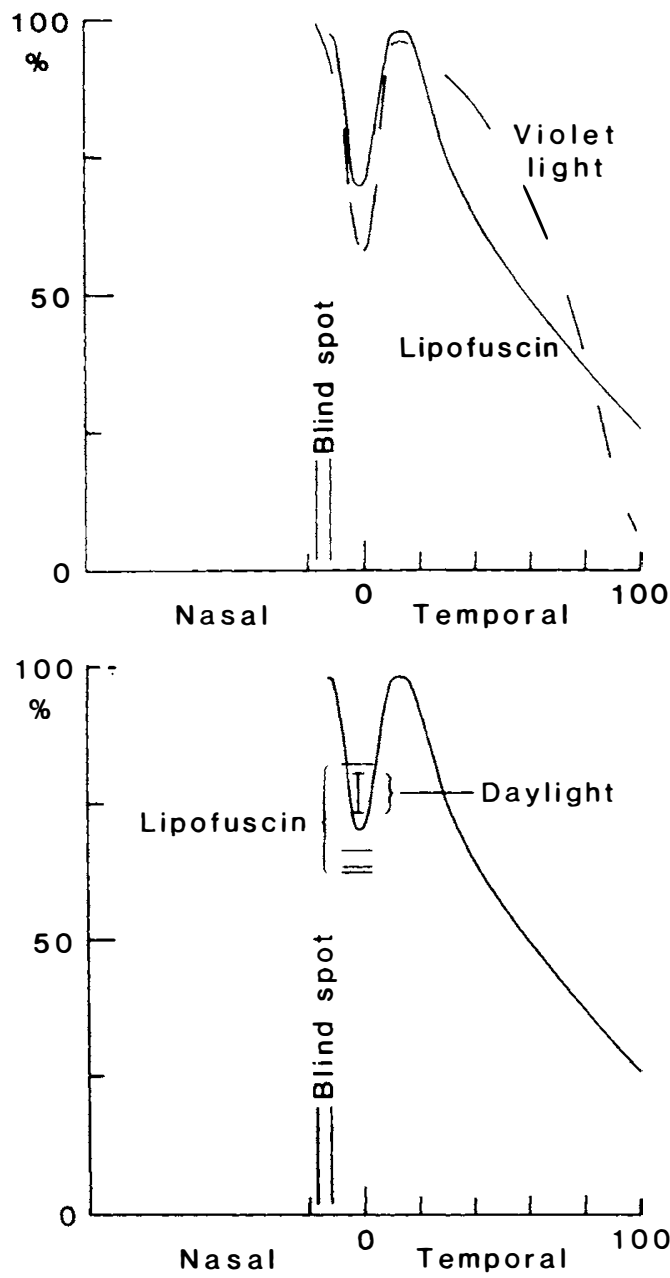

Fig. 2: (a) The distribution of lipofuscin across a principal meridian of the human retina as compared with the calculated distribution of short-wavelength visible radiation; (b) the analogous curve for daylight (the vertical bar indicating the variation due to age), and the variation with age of the accumulation of lipofuscin in the retinal centre for four age groups. (For details see text).

since it does not differ materially from the one for violet light in Fig.2a. The horizontal bars represent observations ${ }^{4}$ for neonates (the top bar) and then, increasingly, age groups of 1.6-30, 31-60, and 61-88 years. The difference between observed and predicted values is just significant $(\mathrm{P}<0.05)$, a result to be received with caution as the component data are not random.

Since the fluorometric probe used in the 
production of these data was far smaller ${ }^{4}$ than the rod-free region of the human retina, the fact that the dip does not drop to zero ${ }^{20}$ might imply that foveal cones are contributing to the generation of this profile. But the potential contribution due to rods can only be ruled out if the study could be extended e.g. to a nyctalopic retina: it is worth noting that rat retinae deprived of photo-receptors either owing to a genetic defect or to photo-toxicity show weaker fluorescence, presumably because their lipofuscin is below normal. ${ }^{23}$

It can also be shown with the aid of eq. 2 that, if the accumulation of lipofuscin were due exclusively to the "blue hazard", the dip would be expected to lie at approximately half the height at which it is, in fact, observed.

But the speed of the initial rise of the accumulation of lipofuscin (Fig. 1) was shown to be incompatible with just unbleached rod and cone photo-pigments being involved. The argument ${ }^{4}$ that the spatial distribution (Fig. 2) of lipofuscin accumulation parallels rod density is therefore unlikely to be of immediate significance. What is more likely to be important is the observation that the density of lipofuscin varies inversely with that of melanin. ${ }^{10}$

Weiter ${ }^{24}$ has recently criticised R W Young's hypothesis ${ }^{25}$ of the photic aetiology of senescent ocular conditions on the ground that the latter determined the distribution of light across the [rat] retina with a relatively small source. As a result of this the macular region was said to appear to be the peak recipient retinal area as regards irradiation, which was one of the factors leading Young to impugn radiation in the aetiology of the conditions he discussed, including senile macular degeneration.

According to Weiter, the use of an extended source would have demonstrated that the retinal irradiation is virtually independent of the perimetric angle. Young's figure showed a marked tailing off toward the ora serrata, simulating Fig. 2, and this would be explained simply, but perhaps a little superficially, on the basis that the pupil subtends a progressively smaller profile area as the viewing angle referred to the optic axis is increased. A limited pupil, just as a limiting filter, will reduce a beam incident on a pupil no matter whether the source is small or extended. But, as Weiter ${ }^{24}$ rightly says, the retinal irradiation is effectively con- stant. The reason, however, is that the solid angle subtended by the diminishing pupil is kept constant by virtue of the retina approaching the aperture due to the curvature of the eyeball (Fig. $3)$. It is important to get this matter into the correct perspective, for if the irradiance is roughly independent of the perimetric angle, then eq. ${ }^{3}$ suggests that melanin, either as an agent or as a protective absorber, can play a role that is a function of its concentration. At least two studies ${ }^{10.26}$ have shown melanin to decrease with age over most of the retinal pigment epithelium with an inverse increase in lipofuscin.

Its progressive loss would help to explain the situation at the centre of the macular area even in the absence of significant amounts of the yellow macular pigments. But it does not directly explain an observation ${ }^{3,4}$ which has been silently overlooked in the past, namely the secondary rise in lipofuscin accumulation that occurs after the age of about 50 years. As far as one can tell, the loss of melanin is monotonic, not diphasic. But, if the early rise in lipofuscin results in some way from the absorption of short-

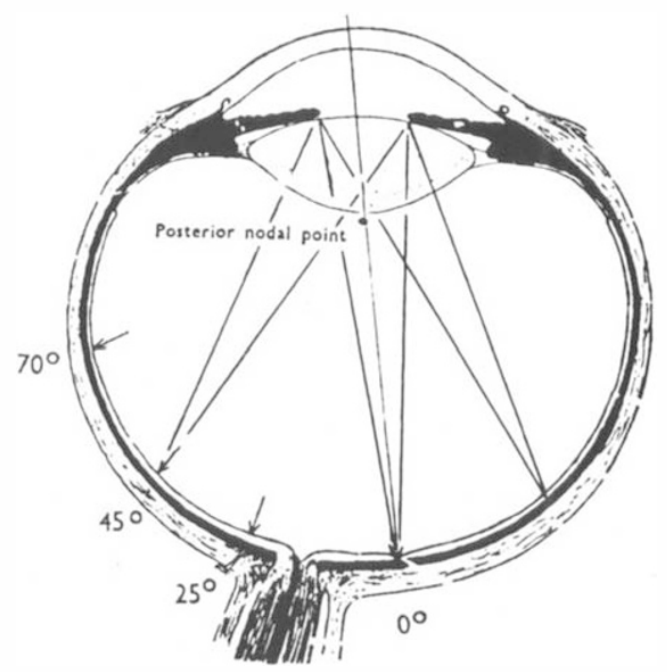

Fig. 3: To show that the curvature of the eye-ball causes the angle of acceptance of radiation to be approximately independent of the perimetric angle over a large part of the retina, indicating the retinal irradiance is not just a function of aperture and filtering effects, but the effective focal distance also has to be taken into account. 
wavelength radiation, should one look in the first place for a different mechanism to explain the second one?

Put another way, if the major part of light to enter our eyes throughout our lifespan does so during the first twenty or so years, and the eye is, as it were, sealed off by the yellowing lens, where is the actinic radiation going to take its second wind from?

The answer may be found in the properties of the crystalline lens. It not only yellows, but it also grows. The yellowing occurs predominately in the nucleus ${ }^{27}$ which occupies approximately one half of the lens in linear dimensions, ${ }^{28}$ i.e. one eighth in volume. ${ }^{16}$ The growth, however, takes place in the relatively transparent cortex (Fig 4). The upshot of this is that, between the ages of twenty-five and fifty years, enough of new cortex has been formed so to increase the circumnuclear space as to provide a marked clearance between the iris and the nucleus. Radiation, not occluded by the eye-brows or the nose, and missing glasses, if worn, will increasingly pass into the retrolental space.

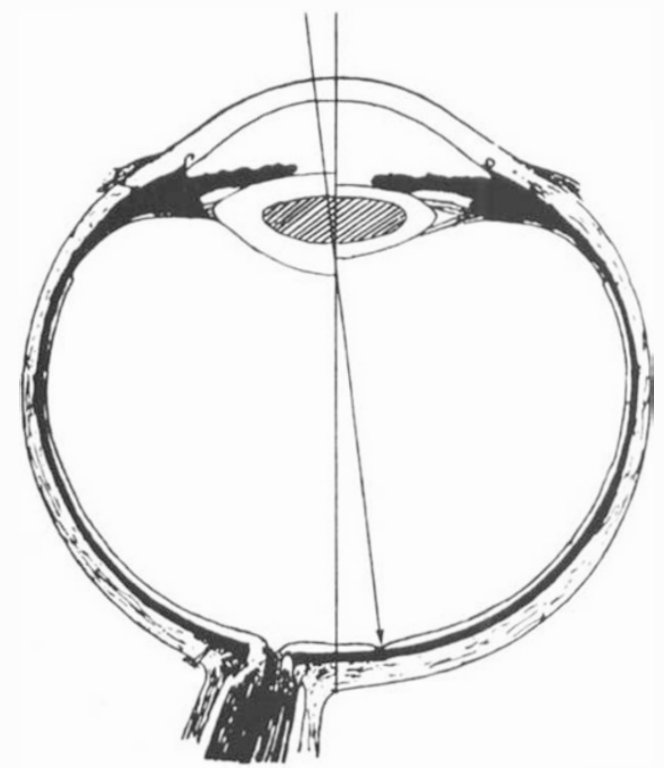

Fig. 4: Section of the eye showing the young lens on the right side of the optic axis, and the old one on the left. Note that the size of the nucleus is unchanged, and that, as a result of lenticular growth, the cortex creates a path between the iris and the nucleus for the passage of short-wavelength radiation into the eye.
Not only that. By virtue of the loss of melanin that has been going on all along, the light striking the inner wall of the eye will be readily reflected. While reflection at the interior of the eye is not all specular, ${ }^{29}$ the geometry of the situation (Fig. 5) makes it likely that a significant fraction reaches the posterior pole. It is possible to put this hypothesis to a test. The visualisation of one's retinal vasculature is rendered feasible either by transillumination of the sclera or by directing a pencil of light into the pupil. But the visualisation of one's optic disc and the vessels branching out therefrom is achieved most readily when the beam introduced through the pupil is directed at the temporal side of the eye: with an undilated pupil, this is the only way of achieving the above aim. This demonstrates that light reflected from the wall of the eyeball avoids being scattered in the lens and reaches the opposite side of the eyeball in such intensity as to render feasible the visualisation of the optic disc. There is no reason to assume that short-wavelength light behaves very differently.

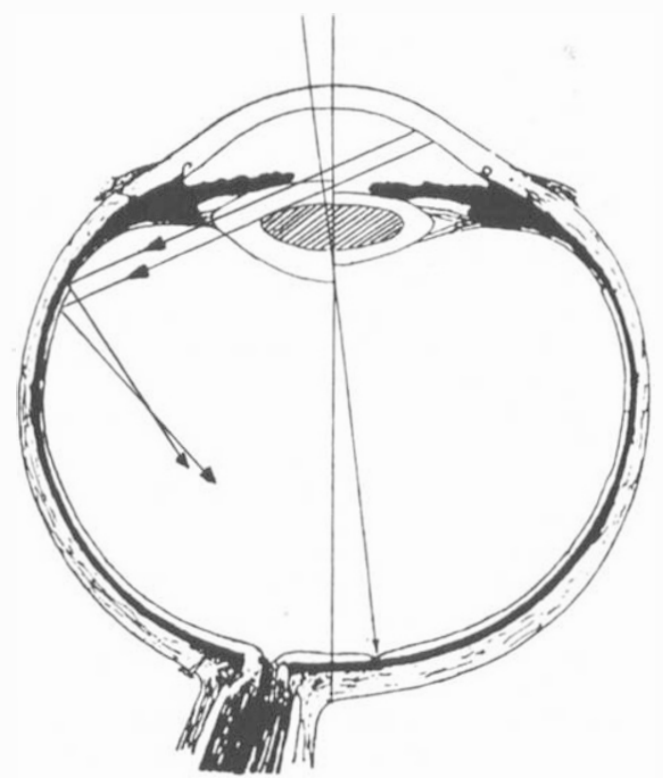

Fig. 5: The radiation admitted into the eye by the mechanism shown in Fig. 3 strikes the near-equatorial belt of the eye interior in such a way as to allow a signficant fraction to be directed at the posterior pole. 


\section{Conclusion}

To conclude, the precise function or raison d'être of lipofuscin is still under discussion; but the conventional wisdom is that "lipofuscin formation and ageing are linked together, probably by oxygen free radicals" and that the pigment "is not believed to be a casual factor in ageing but is regarded as a by-product of cellular reactions which may play a role in the ageing process". ${ }^{30}$ The above analysis supports the view that lipofuscin accumulation in the human retinal pigment epithelium correlates with age within limits because the eyes accumulate light while they clock on the years. A detailed analysis of the factors controlling the intensity of the radiation putatively involved in the formation of lipofuscin throughout our lifespan enables one to reduce the number of explanations of the underlying mechanisms advanced up to the present time.

\section{References}

${ }^{\prime}$ Nandy K and Bourne GH. Effect of centrophenoxine on the lipofuscin pigments in the neurons of senile guinea-pigs. Nature 1966; 210: 313-4.

2 Katz ML, Robinson WG, Herrmann RK, Groome $\mathrm{AB}$, Bieri JG. Lipofuscin accumulation resulting from senescence and Vitimin E deficiency: spectral properties and tissue distribution. Mech Ageing Dev 1984; 25: 149-59.

${ }^{3}$ Feeney-Burns L, Hilderbrand ES, Eldridge S. Ageing human RPE: morphometric analysis of macular, equatorial, and peripheral cells. Invest Ophthalmol Vis Sci 1984; 25: 195-200.

${ }^{4}$ Wing GL, Blanchard GC, Weiter JJ. The topography and age relationship of lipofuscin concentration in the retinal pigment epithelium. Invest Ophthalmol Vis Sci 1978; 17: 601-7.

${ }^{5}$ Katz ML, Robison WG. Nutritional influences on autoxidation, lipofuscin accumulation and ageing. IN: Johnson JE, Walford R, Harman D, Miquel J eds. Free radicals. Ageing, and degenerative diseases. New York: Alan R Liss. 1986: 221-59.

${ }^{6}$ Cutler RG. Evolution of human longevity: a critical overview. Mech Ageing Dev 1979; 9: 337-54.

${ }^{7}$ Aloj Totáro E, Cuomo V, Pisanti FA. Influence of environmental stress on lipofuscin production. Arch Gerontol Geriatr 1986; 5: 343-9.

${ }^{8}$ Katz ML, Robison WG, Dratz EA. Potential role of autoxidation in age changes of the retina and retinal pigment epithelium of the eye. IN: Armstrong $\mathrm{D}$ et al, eds. Free radicals in molecular biology, ageing and disease. New York: Raven Press, 1984: 163-80.

${ }^{9}$ Davies I, Fotheringham A. The effect of lipofuscin on celluar function. Mech Ageing Dev 1983; 23: 347-56.

10) Weiter JJ, Delori FC, Wing GL, Fitch KA. Retinal pigment epithelium lipofuscin and melanin and chorodial melanin in human eyes. Invest Ophthalmol Vis Sci 1986; 27: 145-52.

"Weiter JJ, Delori FC, Wing GL, Fitch KA. Relationship of senile macular degeneration to ocular pigmentation. Am J Opthalmol 1985; 99: 185-7.

12 Lerman S, Borkman R. Spectroscopic evaluation and classification of the normal, ageing and cataractous lens. Ophthalmic Res 1976; 8: 335-53.

${ }_{13}^{13}$ Kagan VE, Shvedova AA, Novikov KN, Kozlov YP. Light-induced free radical oxidation of membrane lipids in photoreceptors of frog retina. Biochim Biophys Acta 1973; 330: 76-9.

${ }^{14}$ Lerman S. Biophysical aspects of corneal and lenticular transparency. Current Eye Res 1984; 3: 3-14.

${ }^{15}$ Lerman S. In vivo and in vitro biophysical studies on human cataractorgenesis. Lens Res 1986; 3: $137-60$.

16 Weale RA. Age and the transmittance of the human crystalline lens. J Physiol (Lond) 1988; 395: 577-87.

17 Weale RA. A biography of the eye. Development - growth - age. London: HK Lewis, 1982.

18 Wyszecki G, Stiles WS. Color Science. Concepts and methods, quantitative data and formulas. New York: Willey \& Sons, 1967.

${ }^{19}$ Ruddock KH. Evidence for macular pigmentation from colour matching data. Vision Res 1963; 3: 417-29.

20) Osterberg G. Topography of the layer of rods and cones in the human retina. Acta Ophthalmol [Suppl] (Copenh) 1935; 6: 1-102.

${ }^{21}$ Sliney DH. Eye protective techiques for bright light. Ophthalmology 1983; 90: 937-44.

22 Katz ML, Eldred GE, Robison Jr GR. Lipofuscin autofluorescence: evidence for vitamin A involvement in the retina. Mech age dev 1987; 39: 81-90.

${ }^{23}$ Katz ML, Drea CM, Eldred GE, Hess HH, Robison Jr WG. Influence of early photoreceptor degeneration on lipofuscin in the retinal pigment epithelium. Exp Eye Res 1986; 41: 561-73.

${ }^{24}$ Weiter, JJ Phototoxic changes in the retina. IN: Clinical light damage to the eye (D. Miller, ed) p.82. London: Springer-Verlag, 1987.

25 Young RW. A theory of central retinal disease. IN: Sears ML. ed. New directions in ophthalmic research. New Haven and London: Yale University Press, 1981: 237-70.

26 Schmidt SY, Peisch, RD. Melanin concentration in normal human retinal pigment epithelium. Invest Opthalmol Vis Sci 1986; 27: 1063-7.

${ }^{27}$ Mellerio J. Yellowing of the human lens: nuclear 
and cortical contributions. Vision Res 1987; 27: ${ }^{29}$ Weale RA. Polarised light and the human fundus 1581-7. oculi. J Physiol (Lond) 1966; 186: 175-86.

${ }^{28}$ Niesel P, Kräuchi H, Bachmann E. Der Abspaltungsstreifen in der Spaltlampenphotographie der alternden Linse. Albrecht von Graefes Arch Klin Exp Ophthal 1976; 199: 11-20.

30 Sohal RS, Wolfe, LS. Lipofuscin: characteristics and significance. Prog Brain Res 1986; 70: 171-83. 